\title{
ON THE ASYMPTOTIC BEHAVIOUR OF EXTREMES AND NEAR MAXIMA OF RANDOM OBSERVATIONS FROM THE GENERAL ERROR DISTRIBUTIONS
}

\author{
R. VASUDEVA, ${ }^{* * *}$ \\ J. VASANTHA KUMARI **** AND \\ S. RAVI, ${ }^{* * * * *}$ University of Mysore
}

\begin{abstract}
As the name suggests, the family of general error distributions has been used to model nonnormal errors in a variety of situations. In this article we show that the asymptotic distribution of linearly normalized partial maxima of random observations from the general error distributions is Gumbel when the parameter of these distributions lies in the interval $(0,1)$. Our result fills a gap in the literature. We also establish the corresponding density convergence, obtain an asymptotic distribution of the partial maxima under power normalization, and state and prove a strong law. We also study the asymptotic behaviour of observations near the partial maxima and the sum of such observations.
\end{abstract}

Keywords: Extremes; general error distribution; Gumbel distribution; strong law for partial maxima; near maxima; power normalization

2010 Mathematics Subject Classification: Primary 60F05

Secondary 60F15; 60G70; 62E20

\section{Introduction}

The class of general error distributions (GEDs) which includes the normal distribution was introduced by Subbotin (1923). This class was popularized by Box and Tiao (1962), (1964), (1973), who used it in robustness studies, and Tiao and Lund (1970), Swamy and Mehta (1977), West (1984), and Osiewalski and Steel (1993). The probability density function (PDF) of a standard GED is given by

$$
f(x)=\frac{v \exp \left\{-(1 / 2)|x / \lambda|^{v}\right\}}{\lambda 2^{1+1 / v} \Gamma(1 / v)}, \quad-\infty<x<\infty,
$$

with $v>0, \lambda=\left(2^{-2 / v} \Gamma(1 / v) / \Gamma(3 / v)\right)^{1 / 2}$, and $\Gamma(\cdot)$ denoting the gamma function. When $v=2$, the GED reduces to the standard normal distribution and, when $v=1$, it reduces to the double exponential distribution. One may trivially see that $\mathbb{E} X^{k}=0$ when $k$ is odd and

$$
\mathbb{E} X^{k}=\left(\frac{\Gamma(1 / v)}{\Gamma(3 / v)}\right)^{k / 2} \frac{\Gamma((k+1) / v)}{\Gamma(1 / v)}
$$

when $k$ is even. This class of distributions has been widely used in statistical modelling. Peng et al. (2009) established that $1-F(x)=F(-x) \sim x^{1-v} f(x)$ as $x \rightarrow \infty$, where $F$ is the

Received 13 December 2012; revision received 21 May 2013.

* Postal address: Department of Studies in Statistics, University of Mysore, Mysore 570006, India.

** Email address: sudeva.rasbagh@ gmail.com

*** Email address: vasanthakumarisj@gmail.com

**** Email address: ravi@ statistics.uni-mysore.ac.in 
distribution function (DF) of the GED. Here ' $\sim$ ' means 'asymptotically equal to' as $x \rightarrow \infty$. One can see that the tail of the GED is asymptotically equal to the Weibullian tail.

Nelson (1991) developed a market volatility model with the GED as the underlying distribution, instead of a normal distribution. Let $\xi_{t}, t \geq 1$, denote the prediction error at time $t$, and let $\sigma_{t}^{2}$ be the variance of $\xi_{t}$, given the information up to time $t$. Nelson introduced the model $\xi_{t}=\sigma_{t} Z_{t}, t \geq 1$, where $\left\{Z_{t}, t \geq 1\right\}$ is a sequence of independent and identically distributed (i.i.d.) random variables (RVs) having the GED as the common distribution. For a data set on daily returns from CRSP (Center for Research in Security Prices-US stock market), over the period from July 1962 to December 1987, it has been shown that a GED with $v=1.58$ (thicker than a normal tail) is a fairly good fit. As a result, one may observe that the conditional distribution of the prediction error $\xi_{t}$ is a GED with the same $v$.

Do and Vetterli (2002) used the GED to model the distribution of wavelets. For experimental results from 640 different data sets on texture images, it was found that GEDs with $v$ ( $\beta$ in their paper) ranging from 0.5 to 1 fit quite well.

If $\left\{X_{n}, n \geq 1\right\}$ denotes an i.i.d. sequence of RVs defined over a common probability space, with the GED as the common DF, certain characteristics of interest are the asymptotic behaviour of the partial sums, partial maxima, etc. Since all the moments exist, one can trivially see that the central limit theorem and the strong law of large numbers hold.

In this paper we discuss the behaviour of maximal errors, as it is equally important. Given a sequence $\left\{X_{n}, n \geq 1\right\}$ of i.i.d. RVs having the GED as the common distribution, define $M_{n}=\max \left\{X_{1}, X_{2}, \ldots, X_{n}\right\}, n \geq 1$. If there exist sequences $\left\{a_{n}, n \geq 1\right\}$ of positive constants and $\left\{b_{n}, n \geq 1\right\}$ of real constants such that $\left(\left(M_{n}-b_{n}\right) / a_{n}\right)$ converges weakly to a nondegenerate $\mathrm{RV} Y$, then it is well known that $Y$ is either Fréchet or Weibull or Gumbel (see, for example, Galambos (1978)). Peng et al. (2009) showed that $Y$ is Gumbel whenever the parameter $v$ of the GED is greater than 1, and Peng et al. (2010) studied the associated rate of convergence. When the parameter $v$ of the underlying GED belongs to $(0,1)$, we establish in Theorem 2.1 below that $Y$ will again be Gumbel. When $v=1$, the GED reduces to the double exponential $\mathrm{DF}$ and in this case it is well known that $Y$ is Gumbel. Our result for $0<v<1$ thus fills a gap in the study of the weak convergence of $\left\{M_{n}, n \geq 1\right\}$. In Section 2 we further establish the density convergence and obtain a strong limit theorem for $\left\{M_{n}, n \geq 1\right\}$ when $v>0$.

Another characteristic of importance, associated with $M_{n}$, is the near maxima, defined as $K_{n}(a)=\#\left\{j: 1 \leq j \leq n, X_{j} \in\left(M_{n}-a, M_{n}\right]\right\}$, which gives the number of observations among $X_{1}, X_{2}, \ldots, X_{n}$ that fall in the interval $\left(M_{n}-a, M_{n}\right]$ for a specified $a$. For a continuous underlying DF, Pakes and Steutel (1997) studied the near maxima and the sum of the associated observations. Let $r_{F}=\sup \{x ; F(x)<1\}$, and let $\bar{F}=1-F$. Suppose that, for any $a>0$, there exists a constant $\gamma(a), 0 \leq \gamma(a) \leq 1$, such that

$$
\lim _{x \rightarrow \infty} \frac{\bar{F}(x)-\bar{F}(x+a)}{\bar{F}(x)}=\gamma(a) .
$$

In Pakes and Steutel (1997), DFs $F$ satisfying (1.1) were classified as thick tailed when $\gamma(a)=0$, medium tailed when $0<\gamma(a)<1$, and thin tailed when $\gamma(a)=1$. They also established that $K_{n}(a) \stackrel{\mathbb{P}}{\rightarrow} 1$ as $n \rightarrow \infty$ when $\gamma(a)=0,\left\{K_{n}(a), n \geq 1\right\}$ converges to a geometric RV taking values $1,2, \ldots$ when $0<\gamma(a)<1$, and $K_{n}(a) \stackrel{\mathbb{P}}{\rightarrow} \infty$ as $n \rightarrow \infty$ when $\gamma(a)=1$, where $\stackrel{\mathbb{\mathbb { P }}}{\rightarrow}$ ' denotes convergence in probability. For thick-tailed distributions, Li (1999) obtained a necessary and sufficient condition for $K_{n}(a) \rightarrow 1$ almost surely (a.s.) as $n \rightarrow \infty$. For thin-tailed $F$, Pakes (2000) established that there exists a normalizing sequence $\left\{v_{n}, n \geq 1\right\}$ such that $\left\{v_{n}^{-1} K_{n}(a), n \geq 1\right\}$ converges weakly to an exponential distribution. 
Another characteristic associated with $K_{n}(a)$ is the near-maxima sum, defined as $S_{n}(a)=$ $\sum_{j \in T_{n}(a)} X_{j}$, where $T_{n}(a)=\left\{j, 1 \leq j \leq n ; X_{j} \in\left(M_{n}-a, M_{n}\right]\right\}$. Pakes (2004) obtained almost-sure results for the behaviour of $\left\{K_{n}(a), n \geq 1\right\}$ and $\left\{S_{n}(a), n \geq 1\right\}$ when $\bar{F}$ is either regularly varying or Weibullian, and $\mathrm{Hu}$ and $\mathrm{Su}$ (2003) examined their behaviour for medium tailed $F$. Balakrishnan and Stepanov (2005) extended some of these results to upper order statistics. In Sections 3 and 4 respectively we discuss the asymptotic behaviour of $\left\{K_{n}(a), n \geq 1\right\}$ and $\left\{S_{n}(a), n \geq 1\right\}$ when $F$ is a GED.

The study of near maxima has found applications in nonlife insurance mathematics, queues with demanding customers, etc. As the class of GEDs includes distributions with thick, medium, and thin tails, the results established in this paper help in developing suitable statistical models in the areas of nonlife insurance, market volatility, queues, etc.

\section{Limit behaviour of partial maxima}

Lemma 2.1. (Peng et al. (2009).) Let $F$ be a GED with parameter $v$. Then

(i) for $v>1$ and $x>0$,

$$
\frac{2 \lambda^{v}}{v x^{v-1}}\left(1+\frac{2(v-1)}{v} \frac{\lambda^{v}}{x^{v}}\right)^{-1} f(x) \leq \bar{F}(x) \leq \frac{2 \lambda^{v}}{v x^{v-1}} f(x),
$$

where $f(x)=c_{1} \mathrm{e}^{-x^{v} / 2 \lambda^{v}}, x \in \mathbb{R}, c_{1}^{-1}=v^{-1}\left(\lambda 2^{1+1 / v} \Gamma(1 / v)\right) ;$

(ii) for any $v>0$,

$$
\bar{F}(x) \sim c_{2} x^{1-v} \mathrm{e}^{-x^{v} / 2 \lambda^{v}} \text { as } x \rightarrow \infty,
$$

where $c_{2}=\left(\lambda^{1-v} 2^{1 / v} \Gamma(1 / v)\right)^{-1}$.

Theorem 2.1. Let $X_{1}, \ldots, X_{n}$ be random observations from a GED $F$ with $0<v<1$, and let $M_{n}=\max \left\{X_{1}, \ldots, X_{n}\right\}, n \geq 1$. Then

$$
\lim _{n \rightarrow \infty} \mathbb{P}\left(M_{n} \leq a_{n} x+b_{n}\right)=\Lambda(x), \quad x \in \mathbb{R},
$$

where

$$
b_{n}=2^{1 / v} \lambda\left(\log n+\frac{1-v}{v} \log \log n+\log c_{2}+\frac{1-v}{v} \log 2 \lambda^{v}\right)^{1 / v}
$$

with $c_{2}=\left(\lambda^{1-v} 2^{1 / v} \Gamma(1 / v)\right)^{-1}, a_{n}=v^{-1} 2^{1 / v} \lambda(\log n)^{(1-v) / v}, n \geq 3$, and $\Lambda(x)=\mathrm{e}^{-\mathrm{e}^{-x}}$.

Proof. From Lemma 2.1(ii), as $n \rightarrow \infty$, we have

$$
n \bar{F}\left(a_{n} x+b_{n}\right) \sim n c_{2}\left(a_{n} x+b_{n}\right)^{1-v} \exp \left(-\frac{\left(a_{n} x+b_{n}\right)^{v}}{2 \lambda^{v}}\right) .
$$

By Taylor's expansion,

$$
\left(a_{n} x+b_{n}\right)^{v}=b_{n}^{v}+\frac{v a_{n} x}{b_{n}^{1-v}}+c^{\prime} \frac{a_{n}^{2} x^{2}}{b_{n}^{2-v}},
$$

where $c^{\prime}$ is some constant. Hence,

$$
\begin{aligned}
\frac{\left(a_{n} x+b_{n}\right)^{v}}{2 \lambda^{v}}= & \log n+\frac{1-v}{v} \log \log n+\log c_{2}+\frac{1-v}{v} \log 2 \lambda^{v}+\frac{v a_{n} x}{2 \lambda^{v} b_{n}^{1-v}} \\
& +\frac{c^{\prime}}{2 \lambda^{v}} \frac{a_{n}^{2}}{b_{n}^{2-v}} x^{2} .
\end{aligned}
$$


Observe that $v a_{n} / 2 \lambda^{v} b_{n}^{1-v} \rightarrow 1$ and $a_{n}^{2} / b_{n}^{2-v} \rightarrow 0$ as $n \rightarrow \infty$. Consequently,

$$
\exp \left(-\frac{\left(a_{n} x+b_{n}\right)^{v}}{2 \lambda^{v}}\right) \sim \frac{\mathrm{e}^{-x}}{c_{2} n(\log n)^{(1-v) / v} 2^{(1-v) / v} \lambda^{1-v}} .
$$

Also,

$$
\left(a_{n} x+b_{n}\right)^{1-v} \sim 2^{(1-v) / v} \lambda^{1-v}(\log n)^{(1-v) / v} .
$$

From (2.1), (2.2), and (2.3), we can see that $\lim _{n \rightarrow \infty} n \bar{F}\left(a_{n} x+b_{n}\right)=\mathrm{e}^{-x}, x \in \mathbb{R}$, and, hence, $\lim _{n \rightarrow \infty} \mathbb{P}\left(M_{n} \leq a_{n} x+b_{n}\right)=\Lambda(x), x \in \mathbb{R}$.

Remark 2.1. The above proof holds for any $v>0$. As such, Theorem 2.1 holds for all $v>0$, i.e. any GED $F$ belongs to the max domain of attraction of a Gumbel law, denoted by $F \in \mathscr{D}(\Lambda)$.

Remark 2.2. Since $F \in \mathscr{D}(\Lambda)$ for any $v>0$, from Theorem 3.1 of Mohan and Ravi (1993), by taking $\alpha_{n}=b_{n}$ and $\beta_{n}=a_{n} / b_{n}, n \geq 1$, we see that

$$
\lim _{n \rightarrow \infty} F^{n}\left(\alpha_{n}|x|^{\beta_{n}} \operatorname{sgn}(x)\right)=\Phi(x)= \begin{cases}0, & x<0, \\ \mathrm{e}^{-1 / x}, & 0 \leq x,\end{cases}
$$

where $\operatorname{sgn}(x)=1,0,-1$ according to whether $x$ is greater than, equal to, or less than 0 . In other words, $F$ belongs to the max domain of attraction of the Fréchet law $\Phi$ under power normalization.

Our next result establishes the density convergence. If $g_{n}(\cdot)$ denotes the PDF of $\left(M_{n}-b_{n}\right) / a_{n}, n \geq 1$, then $g_{n}(x)=n a_{n} F^{n-1}\left(a_{n} x+b_{n}\right) f\left(a_{n} x+b_{n}\right), x \in \mathbb{R}$. The PDF of a Gumbel law is given by $\lambda(x)=\mathrm{e}^{-x} \mathrm{e}^{-\mathrm{e}^{-x}}, x \in \mathbb{R}$.

Theorem 2.2. The PDF $g_{n}(\cdot)$ of $\left(M_{n}-b_{n}\right) / a_{n}$ converges to $\lambda(\cdot)$ locally uniformly on compact subsets of $\mathbb{R}$.

Proof. From Proposition 2.5 of Resnick (1987), it is enough to show that

$$
\lim _{x \rightarrow \infty} f(x) \int_{x}^{\infty} \frac{1-F(t)}{(1-F(x))^{2}} \mathrm{~d} t=1, \quad x>0 .
$$

For $x>0$, observe that $f(\cdot)$ is nonincreasing and, hence, by Proposition 1.17 of Resnick (1987), the above limiting relation holds.

In the next theorem, we establish a strong law for $\left\{M_{n}, n \geq 1\right\}$. In particular, when $v=2$, $F$ is standard normal and, hence, it is well known that $M_{n} / \sqrt{2 \log n} \rightarrow 1$ a.s. (see, for example, Galambos (1978)).

Theorem 2.3. Let $F$ be the DF of the GED with parameters $v$ and $\lambda$. Then

$$
\lim _{n \rightarrow \infty} \frac{M_{n}}{(2 \log n)^{1 / v}}=\lambda \quad \text { a.s. }
$$

Proof. We establish that $M_{n} / b_{n} \rightarrow 1$ a.s. as $n \rightarrow \infty$, where

$$
b_{n}=2^{1 / v} \lambda\left(\log n-\frac{v-1}{v} \log \log n+\log c_{2}-\frac{v-1}{v} \log 2 \lambda^{v}\right)^{1 / v} .
$$


Since $b_{n} \sim \lambda(2 \log n)^{1 / v}$ as $n \rightarrow \infty$, the theorem then follows trivially. For any $k>0$, by Lemma 2.1(ii) we have

$$
\bar{F}\left(k b_{n}\right) \sim c_{2} k^{1-v} b_{n}^{1-v} \exp \left(\frac{-k^{v} b_{n}^{v}}{2 \lambda^{v}}\right) .
$$

Proceeding as in the proof of Theorem 2.1, we see that

$$
\begin{aligned}
\bar{F}\left(k b_{n}\right) & \sim \frac{c_{2}^{1-k^{v}} k^{1-v} \lambda^{\left(k^{v}-1\right)(v-1)} 2^{\left(k^{v}-1\right)(v-1) / v}(\log n)^{\left(k^{v}-1\right)(v-1) / v}}{n^{k^{v}}} \\
& =\frac{c_{3}(\log n)^{\left(k^{v}-1\right)(v-1) / v}}{n^{k^{v}}}
\end{aligned}
$$

where $c_{3}>0$ is some constant. When $k<1$, let $k^{v}=1-\delta_{1}$ for some $\delta_{1} \in(0,1)$. Then from (2.4) we can find an integer $n_{1}$ such that, for all $n \geq n_{1}$,

$$
\bar{F}\left(k b_{n}\right) \geq \frac{c_{3}}{2} \frac{(\log n)^{(1-v) \delta_{1} / v}}{n^{1-\delta_{1}}} .
$$

Consequently, $\sum_{n} \bar{F}\left(k b_{n}\right)=\infty$ whenever $k<1$. For $k>1$, take $k^{v}=1+\delta_{2}$ for some $\delta_{2}>0$. Then, again, from (2.4) we can find an integer $n_{2}$ such that, for all $n \geq n_{2}$,

$$
\bar{F}\left(k b_{n}\right) \leq \frac{2 c_{3}(\log n)^{(v-1) \delta_{2} / v}}{n^{1+\delta_{2}}} .
$$

In turn $\sum \bar{F}\left(k b_{n}\right)<\infty$ whenever $k>1$. By Theorem 4.4.1 of Galambos (1978), we then obtain

$$
\limsup _{n \rightarrow \infty} \frac{M_{n}}{b_{n}}=1 \text { a.s. }
$$

In order to show that $M_{n} / b_{n} \rightarrow 1$ a.s. as $n \rightarrow \infty$, it is enough to show that, for any given $\varepsilon \in(0,1)$,

$$
\mathbb{P}\left(M_{n}<(1-\varepsilon) b_{n} \text { infinitely often }\right)=0 .
$$

By Lemma 4.3.3 of Galambos (1978), (2.5) holds provided $\sum_{n} \bar{F}\left((1-\varepsilon) b_{n}\right)=\infty$ and $\sum_{n} \bar{F}\left((1-\varepsilon) b_{n}\right) \exp \left(-n \bar{F}\left((1-\varepsilon) b_{n}\right)\right)<\infty$. Taking $k=1-\varepsilon$ in $(2.4)$, and setting $k^{v}=$ $(1-\varepsilon)^{v}=1-\varepsilon_{1}$, we can find an integer $n_{2}>0$ such that, for all $n \geq n_{2}$,

$$
\frac{c_{3}}{2} \frac{(\log n)^{(1-v) \varepsilon_{1} / v}}{n^{1-\varepsilon_{1}}} \leq \bar{F}\left((1-\varepsilon) b_{n}\right) \leq \frac{2 c_{3}(\log n)^{(1-v) \varepsilon_{1} / v}}{n^{1-\varepsilon_{1}}} .
$$

Consequently, $\sum_{n} \bar{F}\left((1-\varepsilon) b_{n}\right)=\infty$. Also, for $n \geq n_{2}$,

$$
\bar{F}\left((1-\varepsilon) b_{n}\right) \exp \left(-n \bar{F}\left((1-\varepsilon) b_{n}\right)\right) \leq \frac{2 c_{3}}{n^{1-\varepsilon_{1} / 2}} \exp \left(\frac{-c_{3} n^{\varepsilon_{1} / 2}}{2}\right),
$$

and, hence, $\sum_{n} \bar{F}\left((1-\varepsilon) b_{n}\right) \exp \left(-n \bar{F}\left((1-\varepsilon) b_{n}\right)\right)<\infty$. By appealing to Lemma 4.3 .3 of Galambos (1978), (2.5) is established. The proof is complete.

Remark 2.3. Theorem 4.4.4 of Galambos (1978) gives a necessary and sufficient condition for the strong convergence of $\left\{M_{n}, n \geq 1\right\}$. However, it requires the exact form of $F$, which is not available for a GED and so we use an alternate method. 


\section{Asymptotic behaviour of near maxima}

The near-maxima RV is defined as $K_{n}(a)=\#\left\{j, 1 \leq j \leq n, X_{j} \in\left(M_{n}-a, M_{n}\right]\right\}$, where $a>0$ is some given constant. We show that, as $n \rightarrow \infty, K_{n}(a) \rightarrow 1$ a.s. when $0<v<\frac{1}{2}$, $K_{n}(a) \stackrel{\mathbb{P}}{\rightarrow} 1$, but $K_{n}(a) \nrightarrow 1$ a.s. when $\frac{1}{2} \leq v<1, K_{n}(a)$ converges to a geometric RV when $v=1$, and $K_{n}(a)$, properly normalized, converges to an exponential RV when $v>1$.

Theorem 3.1. For a GED with parameter $v, K_{n}(a) \stackrel{\mathbb{P}}{\rightarrow} 1$ when $0<v<1, K_{n}(a)$ converges to a geometric $R V$ with values $1,2, \ldots$ when $v=1$, and $K_{n}(a) \stackrel{\mathbb{P}}{\rightarrow} \infty$ when $v>1$.

Proof. With $F$ as the DF of the GED, from Lemma 2.1(ii) we have $\bar{F}(x) \sim c_{2} x^{1-v} \mathrm{e}^{-x^{v} / 2 \lambda^{v}}$ as $x \rightarrow \infty$, where $c_{2}=\left(\lambda^{1-v} 2^{1 / v} \Gamma(1 / v)\right)^{-1}$. Note that the tail $\bar{F}(\cdot)$ is asymptotically Weibullian. Proceeding as in Pakes (2004), we can show that $\lim _{x \rightarrow \infty} \bar{F}(x+a) / \bar{F}(x)=1$ if $0<v<1, \lim _{x \rightarrow \infty} \bar{F}(x+a) / \bar{F}(x)=\mathrm{e}^{-a / 2 \lambda}$ if $v=1$, and $\lim _{x \rightarrow \infty} \bar{F}(x+a) / \bar{F}(x)=0$ if $v>1$. Consequently, from Pakes and Steutel (1997), $F$ is thick tailed with $K_{n}(a) \stackrel{\mathbb{P}}{\rightarrow} 1$ if $0<v<1, F$ is medium tailed with $K_{n}(a)$ converging to a shifted geometric RV taking values $1,2, \ldots$ if $v=1$, and $F$ is thin tailed with $K_{n}(a) \stackrel{\mathbb{P}}{\rightarrow} \infty$ if $v>1$.

In order to investigate the almost-sure behaviour of $K_{n}(a)$, we first present the following results.

Theorem 3.2. (Theorem A of $\mathrm{Li}(1999)$.) It holds that $K_{n}(a) \rightarrow 1$ a.s. as $n \rightarrow \infty$ if and only if

$$
\int_{-\infty}^{\infty} \frac{F(x+a)-F(x-a)}{(1-F(x-a))^{2}} \mathrm{~d} F(x)<\infty .
$$

Theorem 3.3. (Theorem B of Pakes (2004).) For some $\delta>0$, let $F(x+a)-F(x-a)=$ $O\left(x^{-\delta}(\bar{F}(x))\right)$ as $x \rightarrow \infty$ and $-\int_{1}^{\infty} \log (\bar{F}(x)) \mathrm{d} x / x^{1+\delta}<\infty$. Then $K_{n}(a) \rightarrow 1$ a.s. as $n \rightarrow \infty$.

Theorem 3.4. It holds that $K_{n}(a) \rightarrow 1$ a.s. if $0<v<\frac{1}{2}$ and $K_{n}(a) \nrightarrow 1$ a.s. if $\frac{1}{2} \leq v<1$.

Proof. By the mean value theorem, we have $F(x+a)-F(x-a)=2 a f(x+\theta a)$, where $-1 \leq \theta \leq 1$ is some constant. Since $f(x), x>0$, is decreasing, we have $F(x+a)-F(x-a) \leq$ $2 a f(x-a)$. For $0<v<1, f(x) / f(x-a) \rightarrow 1$ as $x \rightarrow \infty$. Hence, we can find an $x_{1}>0$ such that, for all $x \geq x_{1}, F(x+a)-F(x-a) \leq 4 a f(x)=4 a x^{1-v} f(x) / x^{1-v}$. Since $\bar{F}(x) \sim 2 \lambda^{v} x^{1-v} f(x) / v$ as $x \rightarrow \infty$,

$$
F(x+a)-F(x-a)=O\left(\frac{\bar{F}(x)}{x^{1-v}}\right) \quad \text { as } x \rightarrow \infty .
$$

Again using the relation $\bar{F}(x) \sim 2 \lambda^{v} x^{1-v} f(x) / v$ as $x \rightarrow \infty$, for a given $\varepsilon \in(0,1)$, we can find an $x_{2}>x_{1}$ such that, for all $x \geq x_{2}, \bar{F}(x) \geq(1-\varepsilon) 2 \lambda^{v} x^{1-v} f(x) / v$, and in turn that $-\log (\bar{F}(x)) \leq x^{v} / 2 \lambda^{v}-(1-v) \log x-\log \left((1-\varepsilon) c_{2}\right)$. Consequently, for some $c>0$,

$$
\int_{x_{2}}^{\infty} \frac{-\log (\bar{F}(x))}{x^{1-v}} \mathrm{~d} x<c \int_{x_{2}}^{\infty} \frac{\mathrm{d} x}{x^{1-2 v}}<\infty
$$

if $0<v<\frac{1}{2}$. For $1<x<x_{2}, 0<F(x)<F\left(x_{2}\right)<1$ implies that

$$
-\int_{1}^{x_{2}} \frac{-\log (1-F(x))}{x^{1-v}} \mathrm{~d} x<\infty .
$$

Applying Theorem 3.3, we note that $K_{n}(a) \rightarrow 1$ a.s when $0<v<\frac{1}{2}$. 
When $\frac{1}{2} \leq v<1$, proceeding as above, we can find an $x_{3}>0$ such that

$$
\frac{F(x+a)-F(x-a)}{1-F(x-a)} \geq \frac{1}{2 x^{1-v}}, \quad x \geq x_{3} .
$$

By Lemma 2.1(ii), we have $f(x) / \bar{F}(x-a) \sim v x^{v-1} / 2 \lambda^{v}$ as $x \rightarrow \infty$. Consequently, we can find an $x_{4} \geq x_{3}$ such that

$$
\int_{x_{4}}^{\infty} \frac{F(x+a)-F(x-a)}{(\bar{F}(x-a))^{2}} \mathrm{~d} F(x) \geq \frac{v}{\lambda^{v}} \int_{x_{4}}^{\infty} \frac{\mathrm{d} x}{x^{1-v}}=\infty,
$$

and, by Theorem 3.2, $K_{n}(a) \nrightarrow 1$ a.s. as $n \rightarrow \infty$ whenever $\frac{1}{2} \leq v<1$, proving the theorem.

Definition 3.1. (Complete convergence.) A sequence $\left\{\xi_{n}, n \geq 1\right\}$ of RVs is said to converge to a constant $c$ under complete convergence if $\sum_{n} \mathbb{P}\left(\left|\xi_{n}-c\right|>\varepsilon\right)<\infty$ for arbitrary $\varepsilon>0$.

Remark 3.1. Whenever $\xi_{n} \rightarrow c$ under complete convergence, then $\xi_{n} \rightarrow c$ a.s.

For constant $\delta>0$, we establish that $\left(K_{n}(a)-1\right) /(\log n)^{1+\delta} \rightarrow 0$ under complete convergence whenever $0<v<1$.

Theorem 3.5. Let $F$ be the DF of a GED with parameter $v, 0<v<1$. Then, for any $\delta>0$, $\left(K_{n}(a)-1\right) /(\log n)^{1+\delta} \rightarrow 0$ under complete convergence.

Proof. For arbitrary $\varepsilon>0$, we need to show that

$$
\sum_{n=1}^{\infty} \mathbb{P}\left(\frac{\left|K_{n}(a)-1\right|}{(\log n)^{1+\delta}}>\varepsilon\right)<\infty
$$

which holds whenever $\sum_{n=1}^{\infty} \mathbb{P}\left(K_{n}(a)>(\log n)^{1+\delta / 2}\right)<\infty$. With $a_{n}=\left[(\log n)^{1+\delta / 2}\right]+1$, we have

$$
\begin{aligned}
\sum_{n=1}^{\infty} \mathbb{P} & \left(K_{n}(a)>(\log n)^{1+\delta / 2}\right) \\
& =\sum_{n=1}^{\infty} \sum_{k=a_{n}}^{\infty} \int_{-\infty}^{\infty} k\left(\begin{array}{l}
n \\
k
\end{array}\right) F^{n-k}(y-a)(F(y)-F(y-a))^{k-1} \mathrm{~d} F(y) .
\end{aligned}
$$

For $t>1$, let $a(t)=(\log t)^{1+\delta / 2}$ and $a^{-1}(t)$ denote its inverse. Then $a\left(a^{-1}(t)\right)=t$ implies that $a^{-1}(t)=\exp \left(t^{(1+\delta / 2)^{-1}}\right)$. Defining $b(t)=\exp \left(t^{(1+\delta / 4)^{-1}}\right)$ we have $b(t)>a^{-1}(t)$ for large $t$. Hence,

$$
\begin{aligned}
\sum_{n=1}^{\infty} \mathbb{P} & \left(K_{n}(a)>(\log n)^{1+\delta / 2}\right) \\
& \leq \int_{-\infty}^{\infty} \sum_{k=1}^{\infty} \sum_{n=k}^{b(k)} k\left(\begin{array}{l}
n \\
k
\end{array}\right) F^{n-k}(y-a)(F(y)-F(y-a))^{k-1} \mathrm{~d} F(y) \\
& =\int_{-\infty}^{\infty} \sum_{k=1}^{\infty} k(F(y)-F(y-a))^{k-1} \sum_{n=k}^{b(k)}\left(\begin{array}{l}
n \\
k
\end{array}\right) F^{n-k}(y-a) \mathrm{d} F(y)
\end{aligned}
$$


Proceeding as in $\mathrm{Hu}$ and $\mathrm{Su}(2003$, p. 234), we can show that

$$
\sum_{n=k}^{b(k)}\left(\begin{array}{l}
n \\
k
\end{array}\right) F^{n-k}(y-a) \leq b^{2}(k) \frac{F^{k}(y-a)}{(1-F(y-a))^{k-1}} .
$$

By (3.1), we obtain

$$
\begin{aligned}
\sum_{n=1}^{\infty} \mathbb{P} & \left(K_{n}(a)>(\log n)^{1+\delta / 2}\right) \\
& \leq \int_{-\infty}^{\infty} \sum_{k=1}^{\infty} k \frac{(F(y)-F(y-a))^{k-1}}{(1-F(y-a))^{k-1}} F^{k}(y-a) b^{2}(k) \mathrm{d} F(y), \\
& \leq \int_{-\infty}^{\infty} \sum_{k=1}^{\infty} k \frac{(F(y)-F(y-a))^{k-1}}{(1-F(y-a))^{k-1}} \exp \left(2 k^{(1+\delta / 4)^{-1}}\right) \mathrm{d} F(y) .
\end{aligned}
$$

Since $F$ is thick tailed, we have $\lim _{y \rightarrow \infty}(F(y)-F(y-a)) /(1-F(y-a))=0$. Consequently, for a given $\delta_{1}>0$, we can find a $y_{1}$ such that, for all $y \geq y_{1}$,

$$
\frac{F(y)-F(y-a)}{\bar{F}(y-a)}<\mathrm{e}^{-\delta_{1}} .
$$

From the fact that $F$ is continuous with $(F(y)-F(y-a)) / \bar{F}(y-a)<1$ for all $|y| \leq y_{1}$, we can find a $y_{2} \in\left[-y_{1}, y_{1}\right]$ such that, for $|y| \leq y_{1}$,

$$
\frac{F(y)-F(y-a)}{\bar{F}(y-a)} \leq \frac{F\left(y_{2}\right)-F\left(y_{2}-a\right)}{\bar{F}\left(y_{2}-a\right)}=\mathrm{e}^{-\delta_{2}}<1
$$

for some $\delta_{2}>0$. For $y<-y_{1}$, note that

$$
\frac{F(y)-F(y-a)}{\bar{F}(y-a)}<\frac{F\left(-y_{1}\right)}{1-F\left(-y_{1}\right)}=\mathrm{e}^{-\delta_{3}}<1
$$

for some $\delta_{3}>0$. Let $\delta_{0}=\min \left(\delta_{1}, \delta_{2}, \delta_{3}\right)$. Then, from (3.2), (3.3), (3.4), and (3.5),

$$
\begin{aligned}
\sum_{n=1}^{\infty} \mathbb{P}\left(K_{n}(a)>(\log n)^{1+\delta / 2}\right) & \leq \int_{-\infty}^{\infty} \sum_{k=1}^{\infty} k \mathrm{e}^{-\delta_{0}(k-1)} \exp \left(2 k^{(1+\delta / 4)^{-1}}\right) \mathrm{d} F(y) \\
& <\infty
\end{aligned}
$$

Consequently, $\left(K_{n}(a)-1\right) /(\log n)^{1+\delta} \rightarrow 0$ under complete convergence, completing the proof.

Remark 3.2. Note that, by the Borel-Cantelli lemma, (3.6) implies that

$$
\frac{K_{n}(a)-1}{(\log n)^{1+\delta}} \rightarrow 0 \quad \text { a.s., }
$$

and this is consistent with Remark 3.1, that is, complete convergence implies almost-sure convergence. 
In the sequel, we obtain the limit distribution of $\left\{K_{n}(a), n \geq 1\right\}$ when $v>1$. By Theorem 3.1, recall that a GED with $v>1$ is thin tailed. Pakes (2000) obtained the limit distribution of $\left\{K_{n}(a)\right\}$ when the underlying DF is thin tailed. In order to obtain the limit distribution, Pakes (2000) further divided the class of thin-tailed distributions into svelte, lean, and gaunt. We first show that a GED with $v>1$ is svelte. By Assumption B(ii) of Pakes (2000, p. 1106), a DF $F$ is svelte if $R(x)=-\log \bar{F}(\log x)$ is slowly varying as $x \rightarrow \infty$ with index function $\varepsilon(x)$ slowly varying as $x \rightarrow \infty$ and satisfying $\varepsilon(x) R(x) \rightarrow \infty$ as $x \rightarrow \infty$.

Theorem 3.6. The DF F of a GED with $v>1$ is svelte.

Proof. If $F$ is the DF of a GED, by Lemma 2.1(ii), we have $\bar{F}(x) \sim c_{2} x^{1-v} \exp \left(-x^{v} / 2 \lambda^{v}\right)$ as $x \rightarrow \infty$. Hence,

$$
R(x)=-\log \bar{F}(\log x)=\frac{(\log x)^{v}}{2 \lambda^{v}}(1+o(1)) \quad \text { as } x \rightarrow \infty .
$$

In turn, for any $t>0, R(t x) / R(x) \rightarrow 1$ as $x \rightarrow \infty$, or $R(x)$ is slowly varying as $x \rightarrow \infty$. The Karamata representation of $R(x)$ is given by $R(x)=\exp \left(\int_{c}^{\infty}(\varepsilon(y) / y) \mathrm{d} y\right)$ for some constant $c$ and the index function $\varepsilon(x) \rightarrow 0$ as $x \rightarrow \infty$. If $R^{\prime}$ denotes the derivative of $R$, we have

$$
R^{\prime}(x)=\frac{1}{\bar{F}(\log x)} \frac{f(\log x)}{x}=\frac{v}{2 \lambda^{v}} \frac{(\log x)^{v-1}}{x}(1+o(1)) .
$$

Also, the Karamata representation gives $R^{\prime}(x)=R(x) \varepsilon(x) / x$. Hence, from (3.7), we see that $\varepsilon(x)=v(1+o(1)) / \log x$ and $\varepsilon(x) R(x)=v(\log x)^{v-1}(1+o(1)) / 2 \lambda^{v}$ as $x \rightarrow \infty$, which means that $\varepsilon(\cdot)$ is slowly varying, and $\varepsilon(x) \rightarrow 0$ as $x \rightarrow \infty$ and $\varepsilon(x) R(x) \rightarrow \infty$ as $x \rightarrow \infty$. Hence, $F$ is svelte.

Theorem 3.7. If $F$ is the DF of a GED with $v>1$ then, for any $x>0$,

$$
\lim _{n \rightarrow \infty} \mathbb{P}\left(K_{n}(a) \leq x v_{n}\right)=1-\mathrm{e}^{-x},
$$

where

$$
v_{n}= \begin{cases}\exp \left(\frac{a v}{2^{1 / 2} \lambda}(\log n)^{1-1 / v}\right), & 1<v<2, \\ \exp \left(-\sum_{j=1}^{k} \frac{v(v-1) \cdots(v-\overline{j-1})}{j !}\left(\frac{-a}{2^{1 / 2} \lambda}\right)^{j}\right. & \\ \left.\times(\log n)^{1-j / v}\right), & k \leq v<k+1, k \geq 2 .\end{cases}
$$

Proof. Let $\Psi(x)=1 / \bar{F}(\log x), x>0$, and let $\lambda(\cdot)$ be its inverse function. Define $H(x)=\Psi\left(\mathrm{e}^{-a} \lambda(x)\right), x>0$. Since $F$ is svelte, by Theorem 5.2 of Pakes (2000), we note that $v_{n}^{-1} K_{n}(a)$ converges weakly to a unit exponential distribution, where $v_{n}=n / H(n)$. We will now find the form of $H(n)$. By Theorem 5.1 of Pakes (2000), $H(n)$ satisfies the relation $\lim _{n \rightarrow \infty} H(n) p_{n}(x)=\mathrm{e}^{-x}, x>0$, with $p_{n}(x)=\bar{F}\left(b_{n}+a_{n} x(1+o(1))-a\right)$, where $a_{n}=v^{-1} 2^{1 / v} \lambda(\log n)^{(1-v) / v}$,

$$
b_{n}=2^{1 / v} \lambda\left(\log n+\frac{1-v}{v} \log \log n+\frac{1-v}{v} \log 2 \lambda^{v}+\log c_{2}\right)^{1 / v},
$$


and $c_{2}=\left(\lambda^{1-v} 2^{1 / v} \Gamma(1 / v)\right)^{-1}$. It is enough for us to obtain the asymptotic form of $H(n)$, which we obtain from the relation

$$
\lim _{n \rightarrow \infty} H(n) p_{n}(1)=\mathrm{e}^{-1}
$$

We have

$$
\begin{aligned}
p_{n}(1) & =\bar{F}\left(b_{n}+a_{n}(1+o(1))-a\right) \\
& \sim c_{2}\left(b_{n}+a_{n}(1+o(1))-a\right)^{1-v} \exp \left(-\frac{1}{2 \lambda^{v}}\left(b_{n}+a_{n}(1+o(1))-a\right)^{v}\right) .
\end{aligned}
$$

Note that

$$
\left(b_{n}+a_{n}(1+o(1))-a\right)^{1-v} \sim 2^{(1-v) / v} \lambda^{1-v}(\log n)^{(1-v) / v} .
$$

We consider two cases.

Case 1: $1<v<2$. By Taylor's expansion, we obtain

$$
\begin{aligned}
\left(b_{n}+a_{n}(1+o(1))-a\right)^{v} & =b_{n}^{v}\left(1+\frac{a_{n}(1+o(1))-a}{b_{n}}\right)^{v} \\
& =b_{n}^{v}+v\left(a_{n}(1+o(1))-a\right) b_{n}^{v-1}+c^{\prime} \frac{\left(a_{n}(1+o(1))-a\right)^{2}}{b_{n}^{2-v}},
\end{aligned}
$$

where $c^{\prime}$ is some constant. On simplification, we obtain

$$
\begin{aligned}
\exp ( & \left.-\frac{1}{2 \lambda^{v}}\left(b_{n}+a_{n}(1+o(1))-a\right)^{v}\right) \\
& \sim \mathrm{e}^{-1} \exp \left(\frac{a v}{2^{1 / v} \lambda}(\log n)^{(v-1) / v}\right) / c_{2} 2^{(1-v) / v} \lambda^{1-v} n(\log n)^{(1-v) / v} .
\end{aligned}
$$

Hence, by (3.9) and (3.10), we have

$$
p_{n}(1) \sim \frac{\mathrm{e}^{-1}}{n} \exp \left(\frac{a v}{2^{1 / v} \lambda}(\log n)^{(v-1) / v}\right) .
$$

By (3.8), we see that

$$
H(n) \sim n / \exp \left(\frac{a v}{2^{1 / v} \lambda}(\log n)^{(v-1) / v}\right)
$$

which in turn gives

$$
v_{n}=\frac{n}{H(n)} \sim \exp \left(\frac{a v}{2^{1 / v} \lambda}\right)(\log n)^{(v-1) / v} .
$$

As we are interested in the limit distribution of $K_{n}(a) / v_{n}$, we can also take

$$
v_{n}=\exp \left(\frac{a v}{2^{1 / v} \lambda}\right)(\log n)^{(v-1) / v} .
$$

Case 2: $k \leq v<k+1, k=2,3, \ldots$ We have

$$
\left(b_{n}+a_{n}(1+o(1))-a\right)^{v}=b_{n}^{v}\left(1+\frac{a_{n}(1+o(1))-a}{b_{n}}\right)^{v} \text {. }
$$


By Taylor's expansion, we obtain,

$$
\begin{gathered}
\left(b_{n}+a_{n}(1+o(1))-a\right)^{v}= \\
b_{n}^{v}+\sum_{j=1}^{k} \frac{v(v-1) \cdots(v-\overline{j-1})}{j !} b_{n}^{v-j}\left(a_{n}(1+o(1))-a\right)^{j} \\
+\frac{c^{\prime \prime}\left(a_{n}(1+o(1))-a\right)^{k+1}}{b_{n}^{k+1-v}}
\end{gathered}
$$

for some constant $c^{\prime \prime}$. Note that

$$
\begin{aligned}
\sum_{j=1}^{k} & \frac{v(v-1) \cdots(v-\overline{j-1})}{j !} b_{n}^{v-j}\left(a_{n}(1+o(1))-a\right)^{j} \\
& =\sum_{j=1}^{k} \sum_{r=0}^{j} \frac{v(v-1) \cdots(v-\overline{j-1})}{j !} b_{n}^{v-j}\left(\begin{array}{l}
j \\
r
\end{array}\right)\left(a_{n}(1+o(1))\right)^{r}(-a)^{j-r}, \\
& =\sum_{j=1}^{k} \sum_{r=0}^{j} \frac{v(v-1) \cdots(v-\overline{j-1})}{r !(j-r) !} b_{n}^{v-j}\left(a_{n}(1+o(1))\right)^{r}(-a)^{j-r}
\end{aligned}
$$

Hence, (3.9) can be written as

$$
p_{n}(1) \sim q_{1 n} q_{2 n} q_{3 n} q_{4 n},
$$

where

$$
\begin{aligned}
& q_{1 n}=c_{2}\left(b_{n}+a_{n}(1+o(1))-a\right)^{1-v} \mathrm{e}^{-b_{n}^{v} / 2 \lambda^{v}} \\
& q_{2 n}=\exp \left(-\frac{v}{2 \lambda^{v}} b_{n}^{v-1}\left(a_{n}(1+o(1))-a\right)\right), \\
& q_{3 n}=\exp \left(-\frac{1}{2 \lambda^{v}} \sum_{j=2}^{k} \frac{v(v-1) \cdots(v-\overline{j-1})}{j !} b_{n}^{v-j}\left(a_{n}(1+o(1))-a\right)^{j}\right), \\
& q_{4 n}=\exp \left(-\frac{c^{\prime \prime}}{2 \lambda^{v}} \frac{\left(a_{n}(1+o(1))-a\right)^{k+1}}{b_{n}^{k+1-v}}\right) .
\end{aligned}
$$

By (3.10), we can show that

$$
\lim _{n \rightarrow \infty} n q_{1 n}=1 .
$$

On simplification, we can see that

$$
\lim _{n \rightarrow \infty} q_{2 n} \exp \left(\frac{a v}{2^{1 / v} \lambda}(\log n)^{(v-1) / v}\right)=\mathrm{e}^{-1}
$$

and

$$
\lim _{n \rightarrow \infty} q_{4 n}=1 .
$$


We will now obtain the asymptotic form of $q_{3 n}$. Note that, for $k \geq 2$,

$$
\begin{aligned}
\sum_{j=1}^{k} & \frac{v(v-1) \cdots(v-\overline{j-1})}{j !} b_{n}^{v-j}\left(a_{n}(1+o(1))-a\right)^{j} \\
& =\sum_{j=2}^{k} \sum_{r=0}^{j} \frac{v(v-1) \cdots(v-\overline{j-1})}{r !(j-r) !} b_{n}^{v-j}\left(a_{n}(1+o(1))\right)^{r}(-a)^{j-r}, \\
& =\sum_{j=2}^{k} \frac{v(v-1) \cdots(v-\overline{j-1})}{j !}(-a)^{j}(\log n)^{(v-j) / v} 2^{(v-j) / v} \lambda^{v-1},
\end{aligned}
$$

since all the terms with $r \geq 1$ tend to 0 as $n \rightarrow \infty$. Consequently,

$$
q_{3 n} \sim \exp \left(-\sum_{j=2}^{k} \frac{v(v-1) \cdots(v-\overline{j-1})}{j !}\left(\frac{-a}{2^{1 / v} \lambda}\right)^{j}(\log n)^{1-j / v}\right) .
$$

Substituting (3.12)-(3.15) into (3.11) yields

$$
p_{n}(1) \sim \frac{\mathrm{e}^{-1}}{n} \exp \left(-\sum_{j=1}^{k} \frac{v(v-1) \cdots(v-\overline{j-1})}{j !}\left(\frac{-a}{2^{1 / v} \lambda}\right)^{j}(\log n)^{1-j / v}\right) .
$$

Recall that $H(n) p_{n}(1) \rightarrow \mathrm{e}^{-1}$ as $n \rightarrow \infty$ and that $H(n)=n / v_{n}$, To obtain an asymptotic approximation of $H(n)$, we note that

$$
v_{n} \sim \exp \left(-\sum_{j=1}^{k} \frac{v(v-1) \cdots(v-\overline{j-1})}{j !}\left(\frac{-a}{2^{1 / v} \lambda}\right)^{j}(\log n)^{1-j / v}\right) .
$$

As we are interested in the limit distribution of $K_{n}(a) / v_{n}$, we can also take

$$
v_{n}=\exp \left(-\sum_{j=1}^{k} \frac{v(v-1) \cdots(v-\overline{j-1})}{j !}\left(\frac{-a}{2^{1 / v} \lambda}\right)^{j}(\log n)^{1-j / v}\right) .
$$

This completes the proof.

Remark 3.3. As a particular case, for the normal distribution, $v=2$ and

$$
v_{n}=\exp \left(a \sqrt{2 \log n}-\frac{a^{2}}{2}\right) .
$$

\section{Asymptotic behaviour of the near-maxima sum}

Recall that the near-maxima sum is given by $S_{n}(a)=\sum_{j \in T_{n}} X_{j}$, where

$$
T_{n}=\#\left\{j, 1 \leq j \leq n ; X_{j} \in\left(M_{n}-a, M_{n}\right]\right\} .
$$

For example, in the insurance industry, if $M_{n}$ denotes the maximum of $n$ claims, $K_{n}(a)$ gives the number of claims close to $M_{n}$ and $S_{n}(a)$, the sum of such claims. As such, $S_{n}(a)$ turns out 
to be a very important indicator. Pakes (2000) and $\mathrm{Hu}$ and Su (2003) studied the asymptotic behaviour of $S_{n}(a)$ for distributions with $r_{F}=\infty$. In this section we study the behaviour of $S_{n}(a)$ when $F$ is the DF of a GED.

From the definition of $S_{n}(a)$, we have the trivial identity $\left(M_{n}-a\right) K_{n}(a) \leq S_{n}(a) \leq$ $M_{n} K_{n}(a)$, which implies that

$$
\lim _{n \rightarrow \infty} \frac{S_{n}(a)}{M_{n} K_{n}(a)}=1 \quad \text { a.s. }
$$

We have the following results.

Theorem 4.1. Let $\eta_{n}=S_{n}(a) / \lambda(2 \log n)^{1 / v}, n \geq 2$. Then $\eta_{n} \rightarrow 1$ a.s. if $0<v<\frac{1}{2}, \eta_{n} \stackrel{\mathbb{P}}{\rightarrow} 1$ if $\frac{1}{2} \leq v<1$, and $\eta_{n}$ converges weakly to a geometric $R V$ if $v=1$.

Proof. From Theorem 2.3, note that $M_{n} / \lambda(2 \log n)^{1 / v} \rightarrow 1$ a.s. as $n \rightarrow \infty$. Hence, writing

$$
\eta_{n}=\frac{S_{n}(a)}{M_{n} K_{n}(a)} \frac{M_{n}}{\lambda(2 \log n)^{1 / v}} K_{n}(a)
$$

the result follows.

Theorem 4.2. When $v>1, S_{n}(a) /(2 \log n)^{1 / v} \lambda v_{n}$ converges weakly to a unit exponential $R V$.

Proof. Writing

$$
\frac{S_{n}(a)}{\lambda(2 \log n)^{1 / v} v_{n}}=\frac{S_{n}(a)}{M_{n} K_{n}(a)} \frac{M_{n}}{\lambda(2 \log n)^{1 / v}} \frac{K_{n}(a)}{v_{n}},
$$

and applying Theorem 2.3 and Theorem 3.7, the result follows immediately in view of (4.1).

Remark 4.1. From the discussions, it is interesting to note that the class of GEDs is a class of symmetric distributions with all moments finite and that the tail of a GED is asymptotically Weibullian. This class includes DFs that are thick tailed as well as those that are thin tailed. In some sense these distributions are sandwiched between those with rapidly increasing tails and with regularly varying tails.

Pareto distributions are being used in many studies, with applications to stock market data, data on loss due to calamities, insurance claims data, etc. If the data indicate reasons to believe that the observations come from a thick-tailed distribution with finite moments, then GEDs with $v<1$ become useful. In such situations, our study throws light on the behaviour of maxima, near maxima, etc., which are crucial characteristics.

Similarly, if the data indicate that the tail of the associated DF is much thinner than a normal tail, one may be able to choose a GED as a better alternative. In the study of the near maxima RV $K_{n}(a)$, we note that $K_{n}(a) / v_{n}$ converges to a unit exponential RV, under weak convergence, whenever $v>1$. Let $r>1$ be some integer. Define $K_{n}(a)=K_{n, r}(a)$ and $v_{n}(a)=v_{n, r}(a)$ when $v=r$, and note that $K_{n, 2}(a) / v_{n, 2}$ and $K_{n, r}(a) / v_{n, r}, r>2$, both converge to a unit exponential RV. Also, note that $v_{n, r} / v_{n, 2} \rightarrow \infty$ as $n \rightarrow \infty$. This means that a much larger dividing or normalizing sequence is needed to get the same limit distribution when $r>2$ as compared to $r=2$. Consequently, in some sense, $K_{n, r}(a)$ can be seen to be larger than $K_{n, 2}(a)$, eventually. That is, for distributions thinner than normal, the near-maxima RV can be seen to be denser, even though $K_{n, r}(a) \stackrel{\mathbb{P}}{\rightarrow} \infty$ whenever $r>1$. In statistical modelling, if a GED with $v>2$ is found to be more appropriate than a normal distribution, the discussions in this paper may help in understanding and appreciating the situation. 


\section{Acknowledgements}

The research of the first two authors was supported by the Science and Engineering Research Council Project No. SR/S4/MS:585/09 of the Department of Science and Technology, New Delhi, India.

\section{References}

Balakrishnan, N. and Stepanov, A. (2005). A note on the number of observations near an order statistic. J. Statist. Planning Infer. 134, 1-14.

Box, G. E. P. And Tiao, G. C. (1962). A further look at robustness via Bayes's theorem. Biometrika 49, 419-432.

Box, G. E. P. AND Tiao, G. C. (1964). A note on criterion robustness and inference robustness. Biometrika 51, $169-173$.

Box, G. E. P. And Tiao, G. C. (1973). Bayesian Inference in Statistical Analysis. Addison-Wesley, Reading, MA.

Do, M. N. AND VetTerli, M. (2002). Wavelet-based texture retrievel using generalized Gaussian density and KullbackLeibler distance. IEEE Trans. Image Process. 11, 146-158.

Galambos, J. (1978). The Asymptotic Theory of Extreme Order Statistics. John Wiley, New York.

Hu, Z. AND Su, C. (2003). Limit theorems for the number and sum of near-maxima for medium tails. Statist. Prob. Lett. 63, 229-237.

LI, Y. (1999). A note on the number of records near the maximum. Statist. Prob. Lett. 43, 153-158.

Mohan, N. R. ANd Ravi, S. (1993). Max domains of attraction of univariate and multivariate $p$-max stable laws. Theory Prob. Appl. 37, 632-643.

Nelson, D. B. (1991). Conditional heteroskedasticity in asset returns: a new approach. Econometrica 59, 347-370.

OsiewAlski, J. AND STEel, M. F. J. (1993). Robust Bayesian inference in $l_{q}$-spherical models. Biometrika 80, $456-460$.

Pakes, A. G. (2000). The number and sum of near-maxima for thin-tailed populations. Adv. Appl. Prob. 32, $1100-1116$.

PAKes, A. G. (2004). Criteria for convergence of the number of near maxima for long tails. Extremes 7, $123-134$.

Pakes, A. G. And Steutel, F. W. (1997). On the number of records near the maximum. Austral. J. Statist. 39, $179-192$.

Peng, Z., Nadarajah, S. And Lin, F. (2010). Convergence rate of extremes for the general error distribution. J. Appl. Prob. 47, 668-679.

Peng, Z., Tong, B. And Nadarajah, S. (2009). Tail behavior of the general error distribution. Commun. Statist. Theory Meth. 38, 1884-1892.

Resnick, S. I. (1987). Extreme Values, Regular Variation, and Point Processes. Springer, New York.

Subbotin, M. (1923). On the law of frequency of errors. Mat. Sb. 31, 296-301.

Swamy, P. A. V. B. And Mehta, J. S. (1977). Robustness of Theil's mixed regression estimators. Canad. J. Statist. 5, 93-109.

TiaO, G. C. AND Lund, D. R. (1970). The use of OLUMV estimators in inference robustness studies of the location parameters of a class of symmetric distributions. J. Amer. Statist. Assoc. 65, 370-386.

WeSt, M. (1984). Outlier models and prior distributions in Bayesian linear regression. J. R. Statist. Soc. B 46, 431-439. 